\title{
A MESSAGE FERRYING SCHEME WITH DIFFERENTIATED SERVICES
}

\author{
Mooi Choo Chuah \\ Department of Computer Science and Engineering \\ 19 Memorial Drive West, Lehigh University \\ Bethlehem, PA 18015, USA \\ chuah@cse.lehigh.edu
}

\section{ABSTRACT}

Routing in partitioned ad-hoc networks is a challenging problem since end-to-end connectivity may not exist. A Message ferrying (MF) [7] scheme has been proposed recently for non-real-time applications where certain mobile nodes referred to as message ferries are designated to collect and deliver packets. In [11], we have investigated a message ferrying scheme that allows urgent messages with more stringent delay requirement to be delivered earlier than regular messages. In this paper, we analyze a system where these two types of messages arrive according to an arrival distribution and that the buffer sizes at the ferry and the nodes are limited. We also assume that the nodes move and that the urgent/regular messages have different delivery deadlines. We design an elliptical zone forwarding (EZF) scheme for the ferry route. Then, we study how three buffer allocation schemes affect the fraction of delivered urgent messages that meet the deadlines as well as the dropping rates of urgent and regular messages due to buffer contention when used together with the EZF scheme. Our results indicate that the softpartitioning scheme allows more urgent messages to be delivered while keeping the drop rate of regular messages low. Next, we compared the system performance achieved by the EZF scheme with the Ferry Initiated Message Ferry with Nearest Neighbor scheme presented in [14] assuming the FIFO scheme is used for the buffer allocation. Our simulation studies show that the EZF scheme we design achieves better performance than the FFM-NN scheme. The EZF routing scheme combined with the buffer allocation scheme can be used to intelligently disseminate information in military networks.

\section{INTRODUCTION}

The advent of mobile communication technology opened up exciting new dimensions to wireless applications together with a number of challenges. One of the challenges is to control and to maintain the communication links between participating entities as they move around. Wireless ad-hoc networks allow nodes to communicate with each other without any existing infrastructure. These rapidly deployable networks are very useful in many areas such as the battlefields, disaster relief centers etc. Many routing algorithms have been proposed for ad-hoc networks [1],[2],[3]. However, most of these algorithms assume that an end-to-end connectivity between any pair of nodes exists. However, in real life scenarios, node

\author{
Peng Yang \\ Department of Computer Science and Engineering \\ 19 Memorial Drive West, Lehigh University \\ Bethlehem, PA 18015, USA \\ pey204@lehigh.edu
}

mobility, the existence of physical obstacles, limited radio range etc may prevent nodes from communicating with others and result in network partitions. The deployed network may also be sparse because devices need to be deployed over a wide geographical range with limited budget. Thus, more intelligent routing schemes need to be designed to allow information to be disseminated in intermittently connected networks e.g. military networks.

As a result of network partitioning, entities may get isolated from network for an unpredicted amount of time. This raises the question of how information can be disseminated in a constantly disconnected network. Real time routing is difficult for such networks, since it is hard to predict how long the network may be disconnected. However, there are several useful applications that do not require bandwidth or delay guarantees e.g. messaging, emails and file transfer. As long as messages from these applications are eventually and timely delivered even in the presence of temporary network partitions, they are still considered useful especially in the battlefield and disaster relief scenarios.

The existence of network partitioning means new approaches other than the traditional "store-and-forward" routing schemes need to be designed. For these disconnected network scenarios, the network devices need to be able to buffer the messages when they cannot find a route to their destinations and attempt to redeliver such stored messages once connectivity to their destinations are restored. A number of schemes have been proposed so far to provide communications in highly partitioned networks. In [4], the authors propose Epidemic Routing for communication in partitioned networks. This scheme has the advantage of delivering all messages assuming no time and memory constraints. However, the blind-flooding of epidemic routing transmits many redundant messages and thus resulting in huge buffering at each node. This leads to a poor scalability for the scheme. In [5], a modified scheme is proposed to overcome the drawbacks of epidemic routing by exploiting the node mobility statistics. The nodes estimate the probabilities of meeting other nodes in the future and forward messages based on these probabilities. This scheme achieves a higher throughput than the epidemic routing in networks with limited memory. 
However, both schemes may lead to unacceptable transmission delays and low throughput since they wait passively for their chances to re-connect. Instead of being reactive, the authors in [6] take a proactive approach. They propose a scheme where nodes modify its trajectory to deliver the message. This strategy works well provided all nodes have complete knowledge of the locations and movements of all other nodes in the network. It is not easy to extend this model for parallel transmissions of multiple messages in the network.

In [7], the authors propose a Message Ferrying (MF) scheme, where a set of nodes called "message ferries" take the responsibility of carrying messages between disconnected nodes. The authors propose the basic design of the MF scheme and develop a general framework to classify variations of the MF systems.

The concept of Message Ferrying is attractive not only in the common battlefield and disaster recovery scenarios where network partition often occurs, but it is also important in two emerging scenarios: sensor networks for habitat monitoring [8] and remote village communication [9]. A three tiered sensor network architecture has been proposed in [10]. The authors propose using mobile entities (called Data Mules) to collect data from sparse sensor networks. The functionality of Data Mules can be realized using message ferries. The use of message ferries in sensor networks results in power saving and interference reduction since the message ferry can move close to the sensor nodes when it wishes to collect data from or dump data to such nodes. For the case of remote village communication, several data kiosks can be installed at fixed locations in the village where the villagers can drop off their electronic messages. Such messages can be carried via USB sticks transported by motor bikes to big cities.

Even though the messages to be delivered in a message ferry system do not require real-time delivery, it is still useful to provide differentiated delivery services similar to the regular and express mail features offered by the post office. Thus, in [11], we consider the case where messages can be divided into two classes, namely urgent and regular messages. Urgent messages have smaller delay requirements and need to be delivered quickly. In our constrained scenario (CS), the message ferry delivers the urgent messages to the destination node immediately after picking them up from the source node. A methodology for designing the ferry route given such a constraint and the performance evaluation of the proposed method was presented in [11]. However, in [11], we assume that the nodes are fixed in their locations and that messages arrive shortly before the ferry visits a node which is not quite realistic. In addition, we also assume that the buffers available at the nodes and at the ferry are unlimited. In this paper, we investigate the scenario where the nodes move and messages arrive according to a certain probability distribution. We assume the buffers available at the nodes and at the ferry are limited. We investigate how the buffer allocation scheme impacts the satisfactory ratio of urgent messages (i.e. urgent messages that are delivered on time), and the message dropping rates of both the urgent and regular messages as a result of buffer contentions at the ferry, source nodes.

The rest of this paper is organized as follows: in Section II, we describe our network model and the three buffer allocation schemes we investigated. In Section III, we discuss in detail how the ferry travels between the nodes. In Section IV, we describe the simulation model, the traffic pattern and the mobility model. Then, we present and discuss our simulation results in Section V. We discuss our preliminary conclusions and the future work that we intend to explore in Section VI.

\section{NETWORK MODEL}

\section{A. Message Ferrying Scheme}

A message ferrying scheme is a proactive approach for message delivering in disconnected networks [7]. The idea is to introduce one or more mobile devices, called message ferries, whose responsibility is to carry messages between disconnected parts of the network. Other network entities, called regular nodes, act as message sources and sinks. A regular node can be an isolated network node or a cluster-head of an isolated subnet. They have communication needs but are disconnected from each other, and hence rely on the message ferry for message delivery. In [7], the authors discuss five major dimensions on the design of a message ferrying system, namely the mobility of the ferry and regular nodes, the number of ferries, the level of coordination and ferry designation. Clearly, the system behavior, the delivery performance, and hence the optimal strategy, are dependent on the assumptions made on these dimensions.

In [7], the MF scheme is applied to regular nodes that are stationary and a single ferry. The system behavior with different network sizes, and the traffic models are evaluated. It has been shown that message ferry routing can achieve reasonable performance even when the nodes are extremely disconnected. In this paper, we introduce two message types with different QoS requirements, and limited buffers to the model in [7], and investigate how the elliptical zone forwarding 
scheme we designed for the ferry route and the corresponding buffer allocation strategies impact the message ferry system performances.

\section{B. Network Model Framework}

We define two types of messages, namely, urgent messages that have shorter delivery delay deadlines and regular messages that have longer delivery delay deadlines. We assume that regular nodes move according to the random waypoint model. They can only communicate to the ferry but not with each other. We assume that the nodes only use high power links to send short reservation messages to the ferry when they have messages to be delivered. Only when the ferry is within a short distance from a node (which is referred to as the regular transmission range) will the node transfer its generated messages to the ferry. Messages between a communication pair are generated at a constant rate.

\section{BUFFER ALLOCATION SCHEMES}

In this paper, we consider three simple buffer allocation schemes that are normally implemented in routers, namely, the drop-from-front (DFF) scheme, fixed partition (FP) scheme and dynamically-partitioned (DP) scheme. For the DFF scheme, older messages will be dropped to accommodate new messages when the buffers are full. For the fixed partition scheme, $x \%$ of the buffer space is reserved for the urgent messages. For the dynamically-partitioned scheme, the regular messages are allowed to occupy at most (1-x)\% of the buffer space but they can be pre-empted by urgent messages.

\section{FERRY ROUTE DESIGN}

We assume that only a single ferry is present in the system and that this ferry never fails. There are two ferry route schemes that we are interested in exploring. In the first scheme referred to as the earliest deadline (ED) scheme, the ferry maintains a list of nodes that need to be visited either because messages need to be picked up or dropped off. This list is ordered based on the deadlines of the messages (assuming that the reservation message indicates the message deadlines). After the ferry has visited the current node, it picks the node with the earliest deadline to visit next. In our second scheme which we referred to as the Elliptical Zone Forwarding (EZF) scheme, we try to minimize the overall average delay while attempting to maintain a high QoS satisfied ratio for urgent messages (which is defined as the fraction of delivered urgent messages that meet the deadlines). The ferry maintains a list of destinations that need to be visited and another list of reservations that it has received (i.e. nodes with messages that need to be pick up). Currently, in the EZF scheme, we give service priority to those messages that have been picked up over those that are waiting to be picked up. After the ferry has visited a node, it checks its destination list and performs the following computation to decide if there is any node in the reservation list that it can visit before visiting a node in the destination list with the earliest deadline.

Assume that there are 3 nodes in the destination list of the ferry, namely $\mathrm{Y}_{1}, \mathrm{Y}_{2}, \mathrm{Y}_{3}$ and that currently the ferry is at node $\mathrm{X}$. For the destination with the earliest deadline, $\mathrm{Y}_{\mathrm{i}}$, the ferry uses $\mathrm{X}$ and $\mathrm{Y}_{\mathrm{i}}$ as the foci, calculates the delay requirement $R_{i}$ for $Y_{i}$ and use $R_{i}$ as the length of the major axis to construct an ellipse. For example, as shown in Figure 1 the length of $A_{i} B_{i}$ is equal to $R_{i}$. Then, we look for a possible node, $Z$, within the ellipse to visit. This node $\mathrm{Z}$ is chosen as the next node to be visited if we can guarantee that the traveling time for $\mathrm{XZ}+\mathrm{ZY}_{\mathrm{i}}<\mathrm{R}_{\mathrm{i}}$. If such a node exists and the ferry has enough buffer to accommodate its request, the ferry will visit that node. If more than one node exist, then the node with the minimum $\mathrm{XZ}+\mathrm{ZY} \mathrm{Y}_{\mathrm{i}}$ will be chosen to be visited next. Otherwise, the ferry will just visit the next node in the destination list with the earliest deadline. Such an approach is taken in an attempt to reduce the overall message delay while maintaining a high delivery ratio for urgent messages. Note that we assume that all packets that are delivered can meet the deadline requirements.

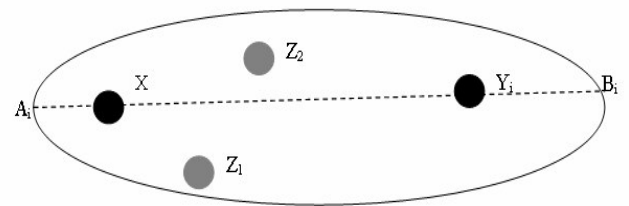

Figure 1: Elliptical Zone Forwarding

\section{Simulation Model}

In this section, we describe the simulation model that we used to evaluate the performance of our designed elliptical zone forwarding scheme when used together with different buffer allocation schemes. We describe the scenario generation, the traffic pattern and the mobility model.

\section{A. Traffic Pattern and Mobility Model}

We use NS-2 [12], which is a network simulation environment designed for wireless ad hoc and sensor networks, as a tool to evaluate our protocols. In our experiments, the MAC layer is implemented using the distributed coordination function of IEEE 802.11 [13]. Messages are sent using CSMA/CA mode. The radio model is consistent with the existing commercial Wireless LAN offerings (e.g. Cisco Aironet). The regular radio transmission range is 250 meters and the channel capacity is $2 \mathrm{Mbit} / \mathrm{sec}$. The simulation area is $5000 * 5000$ 
meter square. Fifty nodes are distributed randomly over this simulation area. The locations of the nodes are chosen such that they are far away from one another and hence can only communicate via the message ferry.

Each simulation lasted for 10,000 seconds of simulation time. The ferry speed is fixed at $15 \mathrm{~m} / \mathrm{s}$. The buffer size is varied from 100 to 600 messages.

\section{B. Traffic Pattern and Mobility Model}

We assume that there are 25 randomly chosen communication pairs where there are 10 pairs of urgent flows and 15 pairs of regular flows. Traffic model is Constant Bit Rate and each message constitutes one UDP packet, and the size of each message is 512 bytes. The interarrival time of the messages on each source/destination pair is 20 seconds.

The mobility model used in our simulation is the random waypoint model described in [3]. The nodes move towards a randomly picked destination at a constant speed. Once the destination is reached, another destination will be randomly chosen and the node will start moving towards the new destination after a certain pause time. This behavior is repeated for the whole duration of the simulation. In our simulation, the node's speed is chosen uniformly between zero and $5 \mathrm{~m} / \mathrm{s}$.

\section{PERFORMANCE EVAluAtion}

To evaluate the performance of our approaches, we use the following metrics:

- Message delivery ratio - the ratio of the number of delivered messages to the number of messages sent by the sources. We compute the overall message delivery ratio (consider both urgent and regular messages together) and the individual urgent or regular message delivery ratio.

- Average End-to-End Delay - the time taken from when the source generates the message to when the destination receives it.

All these metrics reflect the efficiency of the ferry route design and the buffer allocation scheme. A good ferry route design and buffer allocation scheme should have not only a high overall message delivery ratio but also a high QoS satisfied ratio for urgent messages, and low average end-to-end delay. However, a scheme that provides a high QoS satisfied ratio may have to settle for a slightly lower overall message delivery ratio since the regular messages may be dropped to make room for the urgent messages.

The values for the simulation parameters are tabulated in Table 1.

\begin{tabular}{|c|c|}
\hline Parameter & Value \\
\hline Simulation area & $5000 \times 5000 \mathrm{~m}^{2}$ \\
\hline Number of nodes & 50 \\
\hline MAC & 2Mb2.11 DCF \\
\hline Bandwidth & UDP/CBR \\
\hline Traffic pattern & 20 random pairs(10 pairs of high- \\
\hline Traffic load & $\begin{array}{c}\text { priority flows and 15 pairs of low- } \\
\text { priority flows })\end{array}$ \\
\hline Message interval & 20sec \\
\hline Delay requirement & $\begin{array}{c}2000 \text { sec (high-priority message) } \\
3000 \text { sec (low-priority message) }\end{array}$ \\
\hline Buffer Size & $100,200,300,400,500,600$ messages \\
\hline Simulation time & 10000 sec \\
\hline Mobility & $0 \sim 5 \mathrm{~m} / \mathrm{sec}$ \\
\hline Mobility model & Random way-point \\
\hline Ferry speed & $250 \mathrm{~m}$ \\
\hline Transmission range &
\end{tabular}

Table 1: Simulation Parameters

\section{A. Impact of buffer allocation scheme}

In our first experiment, we study the overall message delivery ratio, the urgent/regular message delivery ratio, and the message delay when different buffer allocation schemes are used. We set the deadlines for urgent and regular messages to be $2000 \mathrm{~s}$ and $3000 \mathrm{~s}$ respectively. $\mathrm{x}$ is set to 0.5 for the FP and DP schemes.

Fig. 2 plots the overall message delivery ratio as a function of buffer size for the three buffer allocation scheme. The individual message delivery ratios for urgent and regular messages are plotted in Figs $3 \& 4$. As we can see in Fig. 2, the DFF scheme achieves the best overall delivery ratio but the DP scheme achieves higher delivery ratio for urgent messages as can be seen from Fig 3. This is at the expense of the delivery ratio for regular messages as shown by the plots in Fig 4 . The DFF scheme achieves a smaller average delay because newer messages replace old messages when buffers are full.

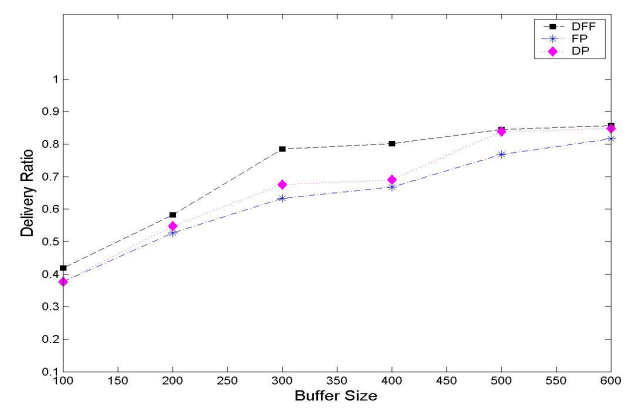

Figure 2.Overall Delivery Ratio vs Buffer Size

We also simulate another scenario where there is only a single pair of high priority traffic with an urgent message arrival rate of 1 message/4 seconds, and 15 pairs 
of low priority traffic with a regular message arrival rate of 1 message/40 seconds. The percentage of urgent messages to the total messages arrived is still maintained at $40 \%$. Thus, $x$ is set at 0.4 for the FP and DP schemes. The overall delivery ratio, the delivery ratios for the urgent and regular messages are plotted in Figures 5, 6 \& 7 respectively. The results indicate that the DP scheme achieves the best overall delivery ratio and allow the urgent messages to achieve $90 \%$ delivery ratio with a buffer size of 500 messages at each regular node.

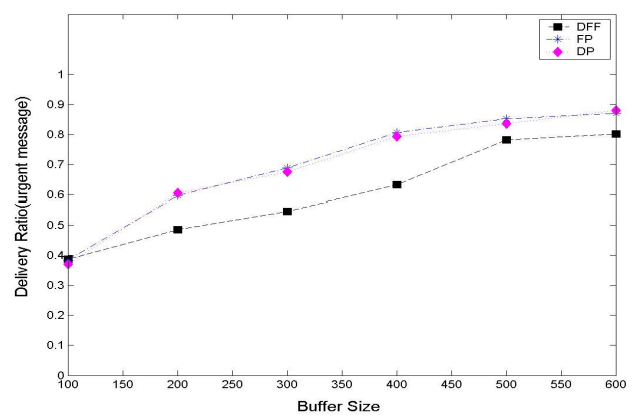

Figure 3: Message Delivery Ratio for Urgent Messages

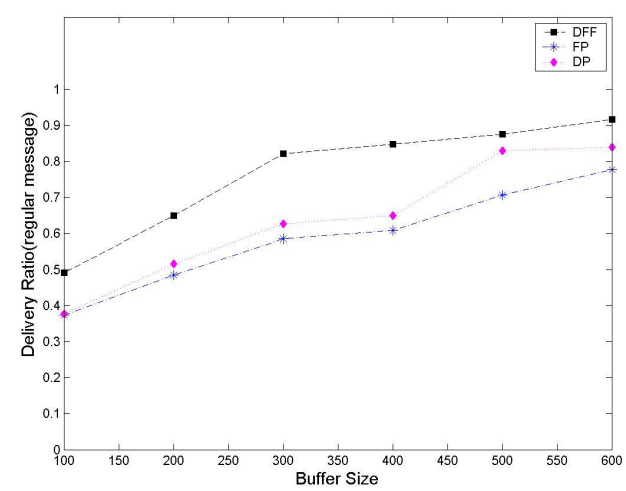

Figure 4: Message Delivery Ratio for Regular Messages

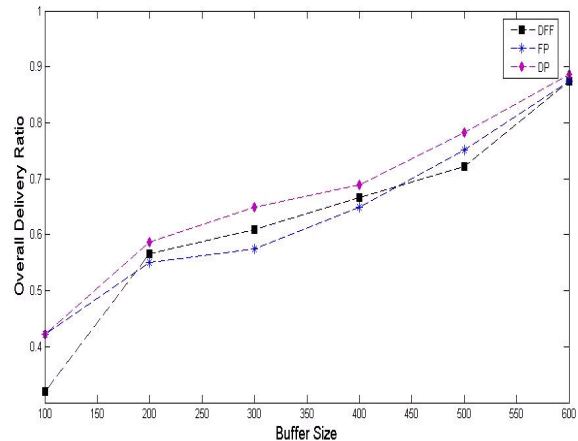

Figure 5: Overall Delivery Ratio (Single Urgent Pair Scenario)

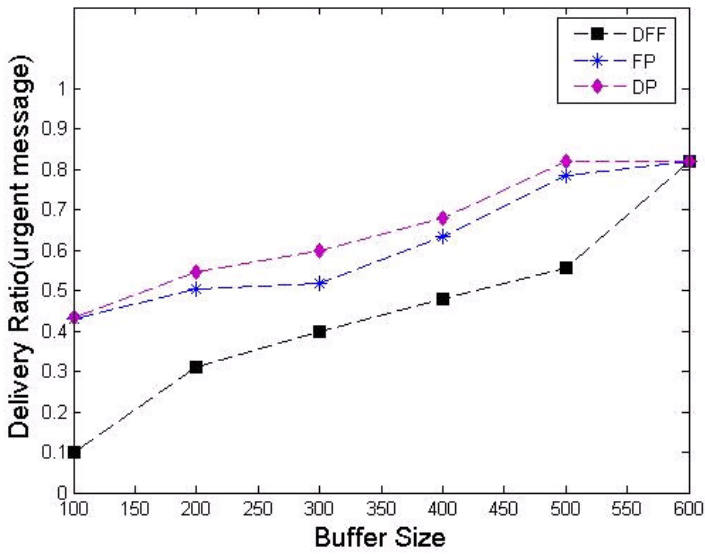

Figure 6: Delivery Ratio for Urgent Messages (Single Urgent Pair Scenario)

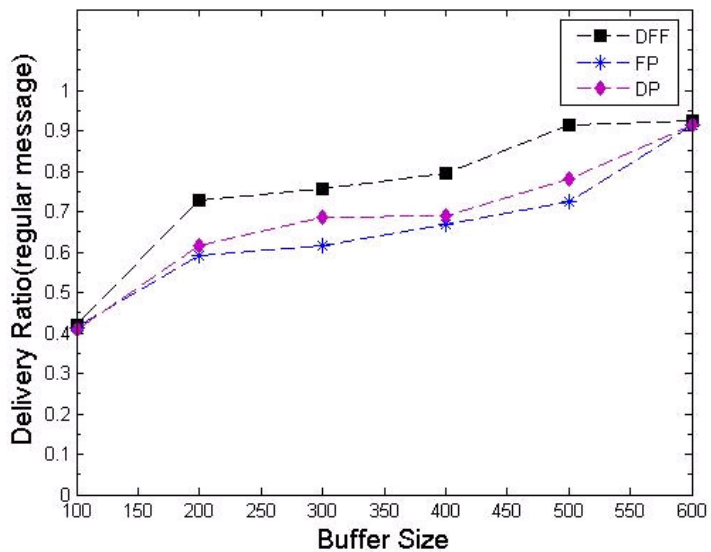

Figure 7: Delivery Ratio for Regular Messages (Single Urgent Pair Scenario)

\section{B. Comparison of different ferry route designs}

In our second experiment, we compare the elliptical zone forwarding (EZF) scheme with the ferry-initiated message ferrying with nearest neighbor first (FFM-NN) scheme described in [14]. In the FFM-NN scheme, the ferry will choose the nearest neighbor to visit next. We implemented the FFM-NN scheme in our NS simulator so that we can compare its performance with our EZF scheme.

In Fig.8, we plot the overall message delivery ratio versus buffer size for the two schemes using the Drop-from-front buffer management strategy. Our results indicate that the EZF scheme gives better overall message delivery ratio than the FFM-NN scheme. In Figs. $9 \& 10$, we plot the delivery ratios of urgent and regular messages versus buffer size while in Fig. 11, we plot the overall average message delay versus buffer size when the two ferry route schemes are used. From Figs. 9 
\& 10, we see that the EZF scheme achieves a higher delivery ratio for both urgent and regular messages when compared to those achieved using the FFM-NN scheme. The EZF scheme also provides a lower overall average message delay when compared to the FFM-NN scheme. The improvement in delay and delivery ratio in the EZF scheme is due to the fact that this scheme serves packets with earliest deadline first. We also have results for the average individual message delay for urgent and regular messages for both schemes. Due to space limitations, we do not include them here. Our results indicate that the EZF scheme provides lower average message delay for both urgent and regular messages when compared to those achieved using the FFM-MN scheme.

\section{Impact of Reservation Ratio}

In this experiment, we fixed the buffer size to 400 messages and varied the reservation ratio for urgent messages, $x$, to see its impacts on the delivery ratio using the DP scheme. Fig. 12 plots the delivery ratios for both urgent and regular messages when $\mathrm{x}$ is varied from 0.3 to 0.6 . Our results show that when the reservation ratio for urgent message increases, the delivery ratio for urgent messages increases but at the expense of the regular messages. The dynamic partition scheme provides us with the flexibility of providing better services to urgent messages if we so desire. For example, setting $x=0.4$, both classes of messages enjoy the same message delivery ratio but setting $\mathrm{x}=0.5$, the message delivery ratio for urgent messages is 0.8 while that ratio for regular messages is 0.65 .

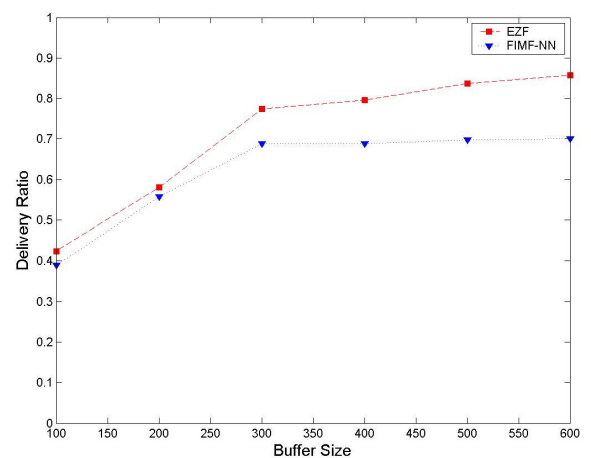

Figure 8. Delivery Ratio vs Buffer Size

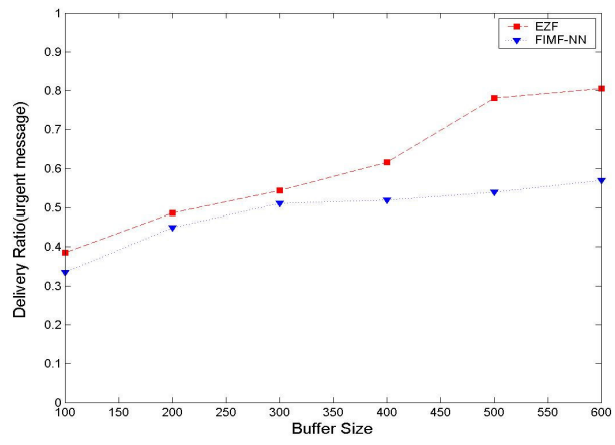

Figure 9. Delivery ratio for urgent messages

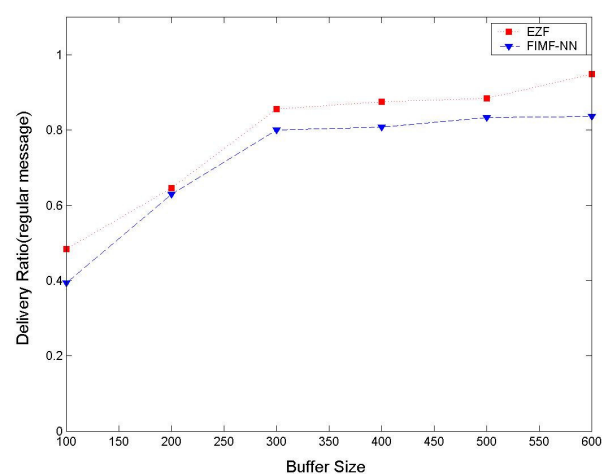

Figure 10: Delivery Ratio for Regular Message

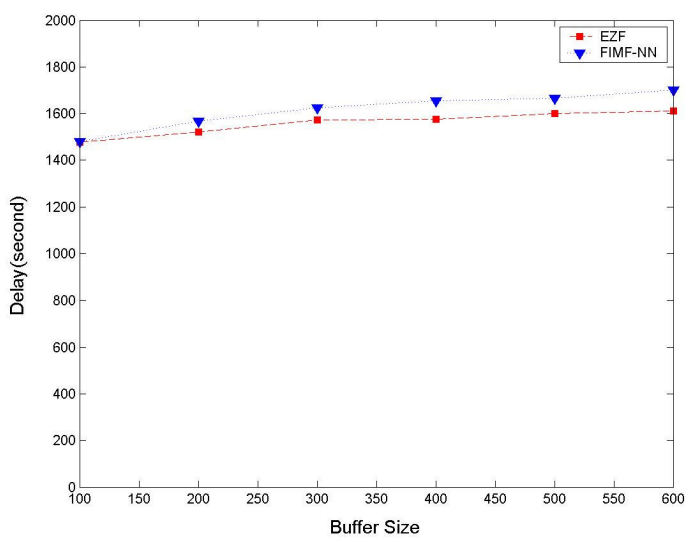

Figure 11. Average Message Delay vs Buffer Size

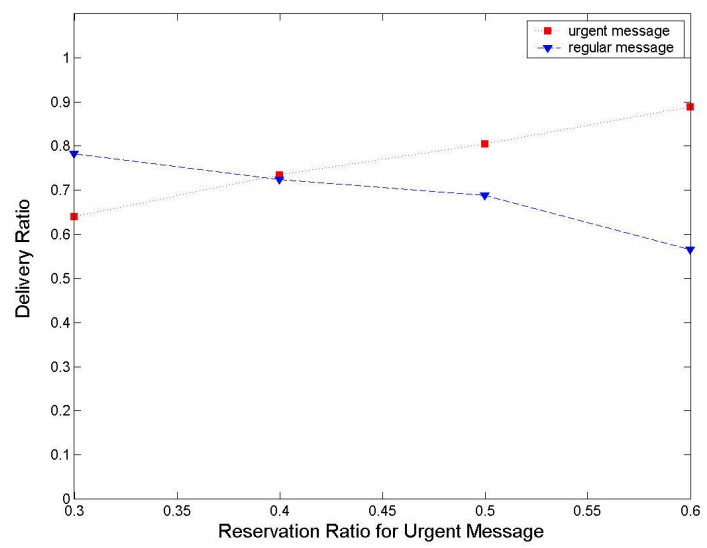

Figure 12: Delivery Ratio vs Reservation Ratio for Urgent Messages.

We also did an experiment where we fixed the regular message arrival rate (1 message/20 second) but varied the urgent message arrival rate as follows: Case 1: $1 \mathrm{msg} / 40 \mathrm{sec}$, Case 2: $1 \mathrm{msg} / 20 \mathrm{sec}$, Case 3: $1 \mathrm{msg} / 15 \mathrm{sec}$, Case 4: $1 \mathrm{msg} / 10 \mathrm{sec}$. The $\mathrm{x}$ value is set to be $0.3,0.5,0.6,0.7$ for the respective cases. Our results (tabulated in Table 2) indicate that the DP scheme can achieve similar overall performance as the DFF scheme with appropriate $\mathrm{x}$ values. 
Table 2: Delivery ratio versus different reservation percentage

\begin{tabular}{|l|r|r|r|r|}
\hline DP Scheme & Case 1 & Case 2 & Case 3 & Case 4 \\
\hline Delivery Ratio (Regular) & 0.707 & 0.672 & 0.553 & 0.514 \\
\hline Delivery Ratio (Urgent) & 0.867 & 0.806 & 0.689 & 0.664 \\
\hline Overall & 0.747 & 0.713 & 0.609 & 0.583 \\
\hline
\end{tabular}

VI. CONCLUSIONS AND FUTURE WORK

In this paper, we investigated how the ferry route design should be done for a message ferrying system where there are two message types, namely urgent and regular messages with different delivery deadlines. We proposed an elliptical zone forwarding (EZF) scheme for delivering urgent and regular messages in such a message ferrying system. We first evaluated how the message delivery ratio for urgent/regular messages are impacted by the choice of buffer allocation schemes. Our results indicate that the dynamic partitioned scheme can achieve a higher delivery ratio for urgent messages at the expense of a slight degradation in the overall message delivery ratio when there are several urgent pairs but the DP scheme achieves the best overall delivery ratio when there is only one urgent pair. Then, we compared the system performance that can be achieved using our EZF scheme with the FFM-NN scheme proposed in [14]. Our results indicate that our EZF scheme can provide a higher overall delivery ratio, higher delivery ratio for urgent messages, lower overall message delay when compared to the FFM-NN scheme. Our EZF routing scheme combined with the DP buffer allocation scheme allow information to be disseminated intelligently among nodes that are outside the transmission range of one another via the message ferry forwarding service. In the near future, we intend to investigate (a) more sophisticated buffer allocation schemes e.g. weighted RED, (b) how different ferry route designs affect the delivery performance. In addition, we also wish to explore the cases where multiple ferries are present. We leave all these interesting topics for our future work.

\section{REFERENCES}

[1] D. Johnson, D. Maltz, "Dynamic Source Routing in Ad-Hoc Wireless Networks", Proceedings of ACM Sigcomm, August 1996.

[2] C. Perkins, E. Royer, "Ad-hoc On-Demand Distance Vector Routing", ACM WoWMoM, Seattle, WA, pp. 26-33, August 1999.

[3] S. Das, C. Perkins, E. Royer, "Performance Comparison of Two Ondemand Routing Protocols", Proceedings of IEEE Infocom, March 2000.

[4] A. Vahdat, D. Becker, "Epidemic routing for partially-connected ad hoc networks", Technical report, Duke University, 2000

[5] J. Davis, A. Fagg, B. Levine, "Wearable computers as packet transport mechanisms in highly-partitioned ad-hoc networks", Proceedings of International Symposium on Wearable Computing, October, 2001.

[6] Q. Li and D. Rus, "Sending messages to mobile users in disconnected ad-hoc wireless networks," Proceedings of Mobicom, August, 1989.

[7] W. Zhao, M. H. Ammar, "Message Ferrying Proactive Routing in Highly-Parititioned Wireless Ad Hoc Networks", Proceedings of the IEEE Workshop on Future Trends in Distributed Computing Systems, Puerto Rico, May, 2003.

[8] P. Juang etc, "Energy-efficient computing for wildlife tracking: design tradeoffs and early experiences with ZebraNet", Proceedings of International Conference on architectural support for programming languages and operating systems (ASPLOS), 2002.

[9] A. Pentland, R. Fletcher, A. Hasson, "DakNet: Rethinking Connectivity in Developing Nations", IEEE Computer Society, 2004.

[10] R. Shah, S. Jain, W. Brunette, "Data MULEs: modeling a 3-tier architecture for sparse sensor networks", Proceedings of Internaitonal Workshop on Sensor Network Protocols and Applications (SNPA), Anchorage, May 2003

[11] R. Viswanathan, J. Li, M. Chuah, "Message Ferrying for Constrained Scenarios", submitted to WoWMoM, 2005

[12] UCB/LBNL/VINT, “The Network Simulator ns-2”, Online at http://www.isi.edu/nsnam/ns/

[13] IEEE Computer Society LAN/MAN Standards Committee, "Wireless LAN Medium Access Protocol (MAC) and Physical Layer Specification", IEEE Std 802.11-1997. The IEEE, New York, NY, 1997.

[14] W. Zhao, M. Ammar, E. Zegura, "A Message Ferrying Approach for data delivery in sparse mobile adhoc networks", Proceedings of Mobihoc, May, 2004

[15] X. Hong, M. Gerla, G. Pei, C. Chiang, "A Group Mobility Model for Ad-hoc Wireless Networks", Proceedings of the 2nd ACM International Workshop on Modeling and Simulatoin of Wirelss and Mobile Systems, 1999. 